\title{
METHODS FOR SOLVING OF INVERSE PROBLEM CONSISTING IN MONITORING FROM SATELLITE OF ARTIFICIAL DISTURBANCES IN THE IONOSPHERE
}

\author{
Sergey N. Zamuruev \\ MIREA-Russian Technological University, http://www.mirea.ru \\ Moscow 119454, Russian Federation \\ zamuruev@mirea.ru
}

Aleksey R. Murlaga

A.I. Berg Central Research Institute of Radioengineering, http://www.cnirti.ru

Moscow 107078, Russian Federation

myrlaga_olga@mail.ru

Abstract. This paper is dealt with control from satellite of artificial disturbances in the ionosphere. Such disturbances can be excited by means of interaction of powerful radiation generated by earthbased heaters with the ionosphere. If next cut-in of a heater, periodicity of cut-ins, parameters of signals radiated by a heater (carrier frequency, modulation frequency, modulation format) are unknown and one has to design an orbit group capable to register primary radiation of a heater or products of its activity then such a problem is suggested to be referred to as inverse problem. A great number of orbit groups differing in parameters come out of solution of inverse problem. To choose the best solution (design of orbit group with optimal parameters) a model function allowing to give an integral description for orbit group and connecting external parameters of orbit group (information being acquired by orbit group from the heater, financial expenditure and period of time being required to deploy the orbit group) is synthesized. External parameters describe orbit group from customer's point of view. External parameters of orbit group are functions of its internal parameters (type and parameters of orbits, quantity of orbits, relative orientation of orbits, number of satellites pro a single orbit and their total number in the orbit group, arrangement of satellites for each orbit). Internal parameters describe orbit group from designer's point of view. Three criterions and appropriate methods for solution of inverse problem consisting in monitoring of earth-based heater are considered in this paper. This criterions and methods take into account the most widespread customer's limitations for external parameters of orbit group. A solution of inverse problem using criterion of heater's minimum non-observation is set forth as an example. This example shows that monitoring of primary radiation generated by a standard heater on conditions that a) one has no limitations in funding and time to deploy an orbit group, b) one can obtain no a priori data about heater's activity and c) one must provide non-stop monitoring of primary radiation generated by a heater, we obtain an orbit group consisting of eight satellites on high-elliptic orbit ("Molniya"-type) with longitude correction in $34^{\circ}$.

Keywords: modification of ionosphere; heater; inverse problem; satellite monitoring

UDC 629.783:551.535

Bibliography - 15 references

Received05.04.2018

RENSIT, 2018, 10(1):23-30

DOI: $10.17725 /$ rensit.2018.10.023

CONTENTS

1. Introduction (24)

2. Formulation of the PRoblem (24)

3. Criterions For SOLUTION OF INVERSE (25) 3.1. Criterion of heater's minimum nonobservation (25)

3.2. Cost criterion (25)
3.3. Criterion «efficiency - cost» (25)

4. Methods FOR SOlytion OF INVERSE PROBLEM (25)

4.1. Method for solution using criterion of heater's minimum non-observation (25)

4.2. Method for solution using cost 
criterion (26)

4.3. Method for solution using criterion «efficiency - cost» (26)

5. A SOLUTION OF INVERSE PROBLEM USING CRITERION OF HEATER'S MINIMUM NONOBSERVATION (26)

6. Resume (28)

7. Conclusion (29)

REFERENCES (29)

\section{INTRODUCTION}

During some last decades ionosphere and magnetosphere are of a wide use in the world to solve a great number of fundamental and applied problems including those from location, remote sensing and communication [1-8].

Some attempts to steer natural processes that take place in the ionosphere and magnetosphere lead to artificial short-time modification of their properties using powerful radio-waves generated by means of earth-based transmitters with subsequent registration of changes thus achieved. For this purposes satellite-based equipment is often used.

Thus, for example, an interaction between coherent very-low-frequency (VLF) waves and high-energy particles in the magnetosphere was under study. A broadband steerable VLF transmitter designed by Stanford University (Siple Station, Antarctica) and navigational VLF transmitter «Omega» (North Dakota, USA) were used as sources of primary radiation. The results of this experiment were registered by means of broadband $(1 \ldots 32 \mathrm{kHz})$ VLF receiver based onboard ISEE-1 satellite [9].

An interaction between magnetospheric electrons of energy from $85 \mathrm{eV}$ to $6.9 \mathrm{keV}$ with VLF radiation generated by earth-based Siple Station was investigated during another experiment. The results of this experiment were registered by means of EXOS-B equipment (broadband $(0.3 \ldots 9 \mathrm{kHz}) \mathrm{VLF}$ receiver and detector of particles $(4 \mathrm{eV}$...6.9 $\mathrm{keV})$ ) [10-11].
DEMETER equipment was used to estimate total power of extremely-low-frequency radiation $(118 \ldots 1197 \mathrm{~Hz})$ injected inside Earthionosphere waveguide previously generated by means of artificial steerable modulation of polar electrojet currents using an earth-based heater [12].

\section{FORMULATION OF THE PROBLEM}

In all examples set forth above a direct problem consisting in monitoring of disturbances in ionosphere or magnetosphere was solved. Key features of this problem are the following [13]:

- next cut-in of earth-based heater is known a priori;

- parameters of a signal radiated by a heater are known a priori;

- effects produced by a heater are being observed by means of a single satellite;

- parameters of an orbit and on-board equipment are being chosen using data known a priori;

- satellite investigations are a pure research (statistical data are collected, there are no requirements for non-stop observation, signal patterns radiated from the ground can be repeated as many times as it is needed).

If next cut-in of a heater, periodicity of cutins, parameters of signals radiated by a heater are a priori unknown and one has to design onboard equipment capable to register primary radiation of a heater or products of its activity (for example, dimensions of a heated spot in the ionosphere, its location and physical properties) then such a problem is suggested to be referred to as inverse problem [14].

Inverse problem as opposed to direct problem may have a lot of solutions each of which is an orbit group with its unique set of the following parameters: total number of satellites in the orbit group, orbit parameters, arrangement of satellites for an orbit. This parameters are suggested to be referred to as internal parameters of the orbit group because they can be varied in the given limits 
during optimization process. On the contrary, external parameters of the orbit group are input data for simulation and cannot be subsequently varied. External parameters describe orbit group from customer's point of view. Internal parameters describe orbit group from designer's point of view. External parameters of orbit group are functions of its internal parameters.

A model function connects external parameters of orbit [14]:

$\Psi(I, F, t)=I^{\alpha} \cdot F^{\beta} \cdot f^{\prime}$,

where $I$ is a function of information being acquired by orbit group from the object of monitoring (the heater); $F$ is a function of financial expenditure being required to deploy the orbit group; $t$ is a function of period of time being required to deploy the orbit group; $\alpha, \beta, \gamma \in[-1 ;+1]$ are constants depending on statement of a problem.

\section{CRITERIONS FOR SOLUTION OF INVERSE PROBLEM}

Different sets of input data and limitations form criterions for simulation [14].

\subsection{Criterion of heater's minimum non- observation}

This criterion is used when an orbit group capable to provide a non-stop monitoring of a heater involving minimum of satellites is required to design. There are no limitations in funding and time to deploy an orbit group. There are no a priori data about heater's activity.

$$
\begin{aligned}
& I\left(q_{\mathrm{j}}, m, d\right)=\mathrm{t}_{\mathrm{n} / \mathrm{obs}}\left(q_{\mathrm{j}}, m, d\right), \\
& \alpha=1, \beta=0, \gamma=0, \\
& \Psi(I, F, t)=t_{\mathrm{n} / \mathrm{obs}}\left(q_{\mathrm{j}}, m, d\right),
\end{aligned}
$$

where $t_{\mathrm{n} / \mathrm{obs}}$ is time of non-observation of a heater; $m$ is number of satellites in the orbit group; $q_{j}$ is $j$ th parameter of an orbit $(j \in[1, \ldots, k]$, where $k$ is a total number of orbit parameters); $d$ defines arrangement of satellites for a given orbit.

Criterion goal consists in finding of such parameters $q_{j}$ and $d$ which give simultaneous minimum of parameter $m$ and model function $\Psi:$

$$
\Psi_{\text {opt }}(I, F, t):\left\{q_{j}, m, d\right\} \rightarrow\left\{\begin{array}{l}
m=\min , \\
\Psi(I, F, t)=0 .
\end{array}\right.
$$

\subsection{Cost criterion}

This criterion is used when an orbit group capable to provide monitoring of a heater with its minimum non-observation (or minimum nonobservation of its activity) involving limitation in funding not exceeding $Y$ is required to design. There are no limitations in time to deploy an orbit group. There are no a priori data about heater's activity.

$I\left(q_{\mathrm{j}}, m, d\right)=\mathrm{t}_{\mathrm{n} / \mathrm{obs}}\left(q_{\mathrm{j}}, m, d\right)$,

$\alpha=1, \beta=0, \gamma=0$,

$\Psi(I, F, t)=t_{\mathrm{n} / \mathrm{obs}}\left(q_{\mathrm{j}}, m, d\right)$,

Criterion goal consists in finding of such parameters $q$, $m$ and $d$ which give $F$ not more than $Y$ while model function $\Psi$ reaches its minimum.

$$
\Psi_{\text {opt }}(I, F, t):\left\{q_{j}, m, d\right\} \rightarrow\left\{\begin{array}{l}
F\left(q_{j}, m\right) \leq Y, \\
\Psi(I, F, t)=\mathrm{min},
\end{array}\right.
$$

where $Y$ is the maximum cost of the orbit group.

\subsection{Criterion «efficiency - cost»}

This criterion is used when with minimum funding one is required to design an orbit group capable to provide monitoring of a heater with its minimum non-observation (or with minimum non-observation of its activity). There are no limitations in time to deploy an orbit group. There are no a priori data about heater's activity.

$I\left(q_{\mathrm{j}}, m, d\right)=\mathrm{t}_{\mathrm{obs}}\left(q_{\mathrm{j}}, m, d\right)$,

$\alpha=1, \beta=-1, \gamma=0$,

$\Psi(I, F, t)=\frac{t_{o b s}\left(q_{j}, m, d\right)}{F\left(q_{j}, m\right)}$,

where $t_{\text {obs }}$ is time of observation of a heater.

Criterion goal consists in finding of such parameters $q, m$ and $d$ which give maximum of model function $\Psi$ :

$\Psi_{\text {opt }}(I, F, t):\left\{q_{j}, m, d\right\} \rightarrow \Psi(I, F, t)=\max$.

\section{METHODS FOR SOLUTION OF INVERSE PROBLEM}

4.1. Method for solution using criterion of heater's minimum non-observation

1. One collects input data for simulation (sighting angles are being defined). Sighting angle is an 
angular distance passing which satellite observes a heater.

A. In case of observing of a heater:

- a beam (or beams) of heater's phased array pattern are being chosen for monitoring;

- a width of a selected beam is being calculated.

B. In case of observing of heater's activity dimensions of ionosphere disturbed due to heater's activity are being defined.

2. For a designed system an orbit is being chosen. Parameters of this orbit are being optimized to provide minimum time of non-observation of a heater by a single satellite.

$$
\left\{q_{\text {jopt }}\right\}:\left\{q_{\mathrm{j}}\right\} \rightarrow t_{\mathrm{n} / \mathrm{obs}}\left(q_{\mathrm{j}}, m=1\right)=\min .
$$

3. Arrangement of satellites for a given orbit is being optimized to provide minimum time of non-observation of a heater by an obit group.

$$
\{m, d\} \rightarrow\left\{\begin{array}{l}
m=\min , \\
t_{n / o b s}\left(q_{\text {jopt }}, m, d\right)=\min .
\end{array}\right.
$$

\subsection{Method for solution using cost criterion}

1. One collects input data for simulation (sighting angles are being defined).

A. In case of observing of a heater:

- a beam (or beams) of heater's phased array pattern are being chosen for monitoring;

- a width of a selected beam is being calculated. B. In case of observing of heater's activity dimensions of ionosphere disturbed due to heater's activity are being defined.

2. For a designed system an orbit is being chosen. Parameters of this orbit are being optimized to provide minimum time of non-observation of a heater by a single satellite.

$$
\left\{q_{\text {jopt }}\right\}:\left\{q_{\mathrm{j}}\right\} \rightarrow t_{\mathrm{n} / \mathrm{obs}}\left(q_{\mathrm{j}}, m=1\right)=\min \text {. }
$$

3. According to limitations in funding maximum number of satellites in the orbit group is being defined.

$$
\left\{m_{0}\right\}:\{m\} \rightarrow\left\{\begin{array}{l}
m=\max , \\
F\left(q_{\text {jopt }}, m\right) \leq Y .
\end{array}\right.
$$

4. Arrangement of satellites for a given orbit is being optimized to provide minimum time of non-observation of a heater by an obit group.

$$
\{\mathrm{d}\} \rightarrow t_{\mathrm{n} / \mathrm{obs}}\left(q_{\mathrm{jopt}}, m_{0}, d\right)=\min \text {. }
$$

\subsection{Method for solution using criterion «efficiency - cost»}

1. One collects input data for simulation (sighting angles are being defined).

A. In case of observing of a heater:

- a beam (or beams) of heater's phased array pattern are being chosen for monitoring;

- a width of a selected beam is being calculated.

B. In case of observing of heater's activity dimensions of ionosphere disturbed due to heater's activity are being defined.

2. For a designed system an orbit is being chosen. Parameters of this orbit are being optimized to provide maximum time of observation of a heater by a single satellite.

$\left\{q_{\text {opt }}\right\}:\left\{q_{\mathrm{j}}\right\} \rightarrow t_{\mathrm{n} / \mathrm{obs}}\left(q_{\mathrm{j}}, m=1\right)=\min$.

3. For each number of satellites in the orbit group arrangement of satellites for a given orbit is being optimized to provide maximum time of observation of a heater by an orbit group.

$\left\{q_{\mathrm{opt}}\right\}:\{m, d\} \rightarrow t_{\mathrm{obs}}\left(q_{\text {jopt }}, m, d\right)=\max$.

4. A function of financial expenditure $F\left(q_{\text {iopt }}, m\right)$ is being synthesized using a priori data.

5. A model function $\Psi(m)$ of the orbit group is being synthesized:

$\Psi(m)=\frac{t_{\text {obs }}\left(q_{\text {jopt }}, m, d_{\text {opt }}\right)}{F\left(q_{\text {jopt }}, m\right)}$.

6. Number of satellites in the orbit group is being defined to provide maximum of the model function:

$m_{0}:\{m\} \rightarrow \Psi(m)=\max$.

\section{A SOLUTION OF INVERSE PROBLEM USING CRITERION OF HEATER'S MINIMUM NON- OBSERVATION}

Let it be required to design an orbit group capable to provide a non-stop monitoring of a primary radiation of a heater involving minimum of satellites. There are no limitations in funding and time to deploy an orbit group. There are no a priori data about heater's activity.

As an object for monitoring we shall choose a standard heater located in auroral region and capable to generate signals of a great effective 


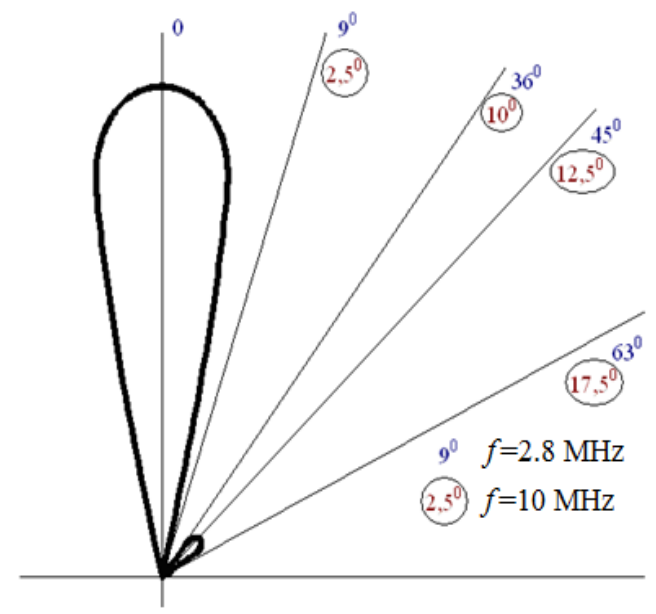

Fig. 1. The radiation pattern of the $\mathrm{FAR}$ radiation of a typical heating stand (main and first side lobe) for the two boundary frequencies of its operating range.

power (up to several GW) in frequency range from 2.8 to $10 \mathrm{MHz}$.

Directivity pattern for a phased array of a standard heater (main beam and first lower beam) for two threshold frequencies of its working range is given on Fig. 1 .

As an orbit for the orbit group we shall choose high-elliptic orbit ("Molniya"-type) (dip angle $i=63.4^{\circ}$; apogee altitude $h_{a}=40112 \mathrm{~km}$; orbit has two circuits 12 hours each) because it is impossible to use geostationary orbit due to high-latitude location of an object under monitoring [15].

According to data from Fig. 1 observation time of a standard heater using a single satellite was calculated. All computations were provided involving numerical algorithm developed in A.I. Berg Central Research Institute of Radioengineering. In our computations $t=5$ was taken as a time step to provide a compromise between accuracy of parameters' definition and data level to process. As a consequence accuracy in definition of periods of observation/nonobservation was \pm 5 minutes.

The result of computations was unsatisfactory (a few time of observation of a heater's primary radiation using a single satellite).

Parameters of the chosen orbit were corrected (a longitude shift in $34^{\circ}$ was provided). As a result we obtained at frequency $f=2.8 \mathrm{MHz}$
SOME METHODS FOR SOLVING OF INVERSE PROBLEM CONSISTING IN MONITORING FROM SATELLITE...

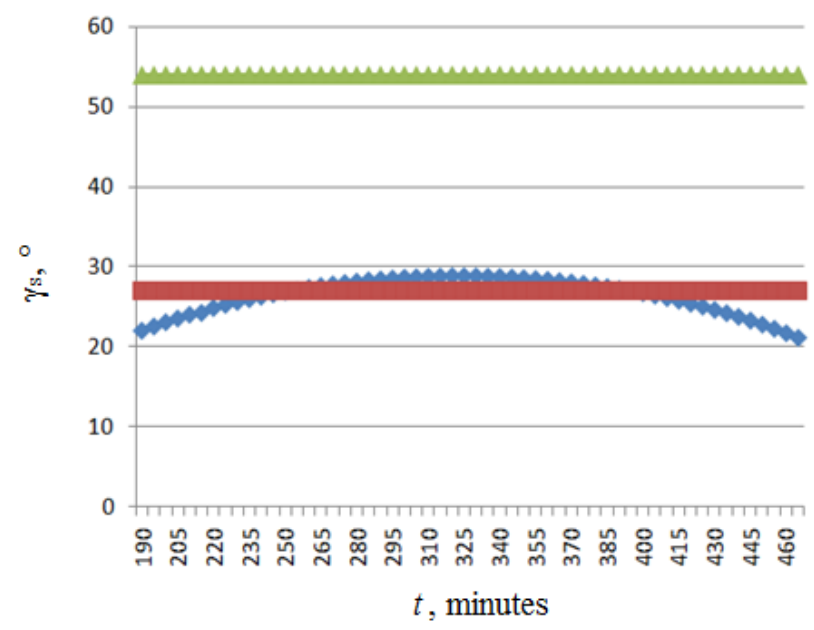

Fig. 2. Observation of a typical heating stand at a frequency $f=2.8 \mathrm{MHz}$ on the first side lobe of its FAS radiation from a satellite located in a highly elliptical Molniya orbit, shifted by longitude by $34^{\circ}\left(\gamma_{s}\right.$ is the viewing angle of the stand).

on the first circuit 145 minutes (or 10.1\% from the time of the whole diurnal cycle) of heater's observation involving first lower beam of its directivity pattern (see Fig. 2) while on the second circuit 410 minutes (or 28.5\% from the time of the whole diurnal cycle) of heater's observation involving main beam of its directivity pattern (see Fig. 3). At frequency $f=10 \mathrm{MHz}$ first circuit is idle while the second circuit acquired at first 35 minutes (or $2.4 \%$ from the time of the whole diurnal cycle) of heater's observation involving first lower beam of its directivity pattern then 230 minutes (or $16.0 \%$ from the time of the whole

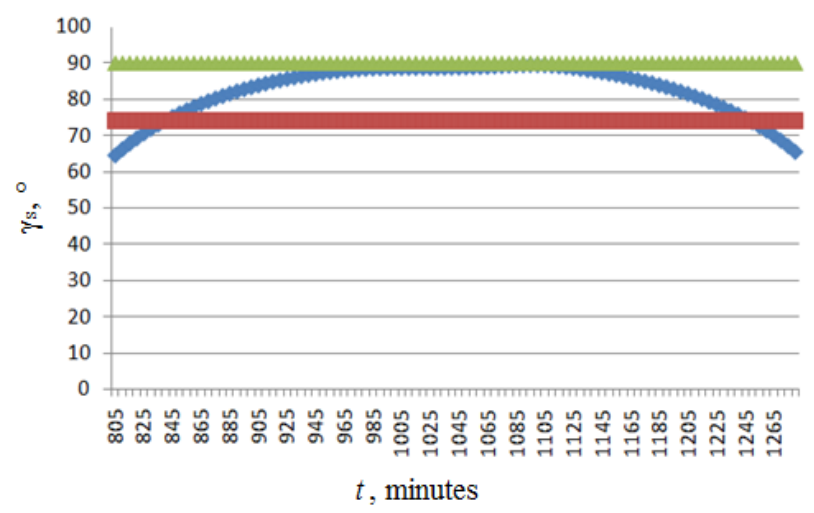

Fig. 3. Observation of a typical heating bench at a frequency $f=2.8 \mathrm{MHz}$ along the main lobe of its $\mathrm{F} A S$ radiation from a satellite located in a highly elliptical Molniya orbit, shifted by longitude by $34^{\circ}$. 


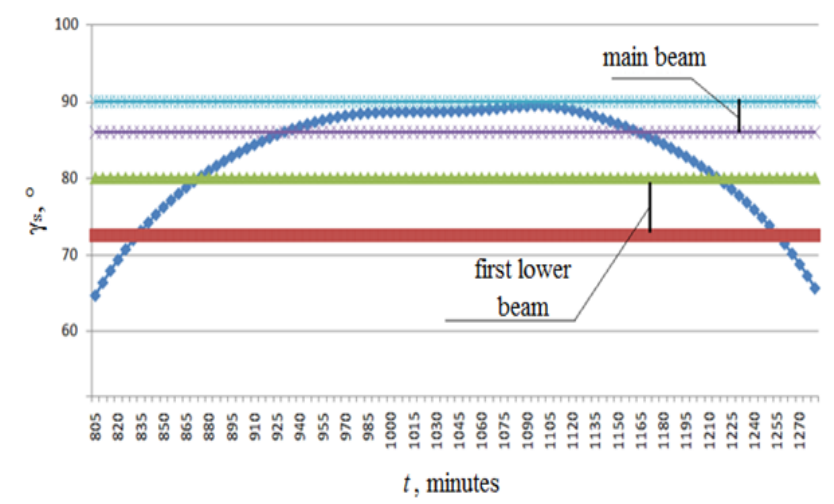

Fig. 4. Observation of a typical heating stand at a frequency $f=10 \mathrm{MHz}$ along the main and first side lobe of its $\mathrm{PHA}$ radiation from a satellite located in a bighly elliptical orbit of the Molniya type, shifted by longitude by $34^{\circ}$.

diurnal cycle) of heater's observation involving main beam of its directivity pattern and at last 45 minutes (or $3.1 \%$ from the time of the whole diurnal cycle) of heater's observation involving first lower beam of its directivity pattern (see Fig. 4).

A mathematical model of orbit group involving timing loop (an imaginary circle the whole arc length of which is 24 hours or 1440 minutes) was constructed. Different arrangement of satellites on the orbit corresponds in this case to a set of periods of time on the timing loop describing a standard heater's observation and non-observation. Some examples of timing loops are shown on Fig. 5.

Optimization made for satellite's arrangement on the orbit gives the following results: $\Psi=0$ for $m=8$ (see Fig. 6).

\section{RESUME}

1. A classification of arbitrary-orbit-group parameters consisting in discrimination between internal and external parameters is suggested. External parameters of orbit group are functions of its internal parameters.

2. To design an orbit group with optimal internal parameters a mathematical method allowing to give an integral description for an orbit group was developed. At the root of the method lies a model function connecting external parameters of the orbit group.

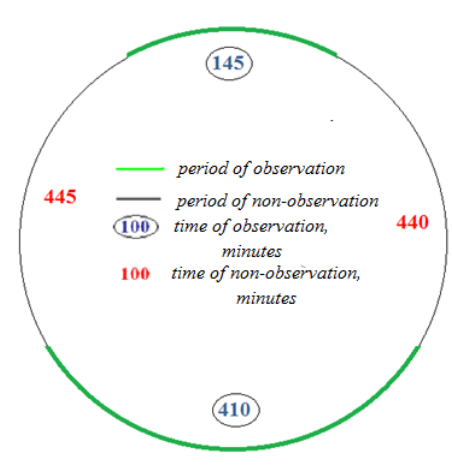

$a$

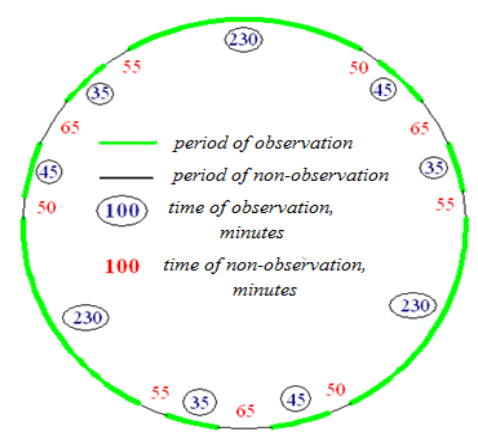

b

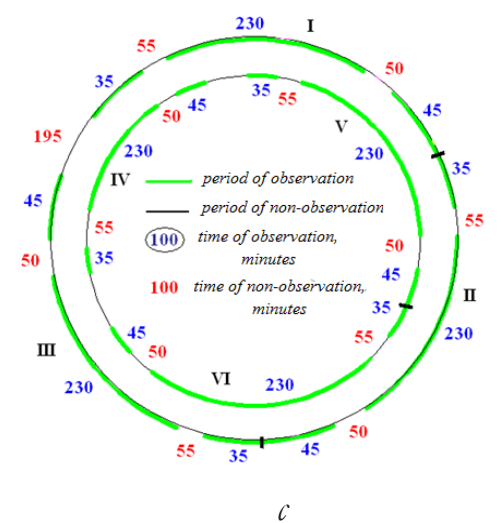

Fig. 5. The time cycle for one satellite monitoring a typical beating stand operating at a frequency $f_{1}=2.8 \mathrm{MHz}$ (a) for three satellites monitoring a typical heating stand operating at a frequency of $f_{2}=10 \mathrm{MHz}$ (b) and for six satellites driving monitoring of a typical heating stand operating at a frequency of $f_{2}=10 \mathrm{MHz}$ (c) Roman numerals indicate the numbers of satellites in the orbital constellation).

3. For practical use of a suggested mathematical method some computational methods were developed which take into account different customer's limitations for external parameters of the orbit group.

4. A solution of inverse problem using criterion of heater's minimum non-observation is set forth as an example. This example shows that monitoring of primary radiation generated 
SOME METHODS FOR SOLVING OF INVERSE PROBLEM CONSISTING IN MONITORING FROM SATELLITE...

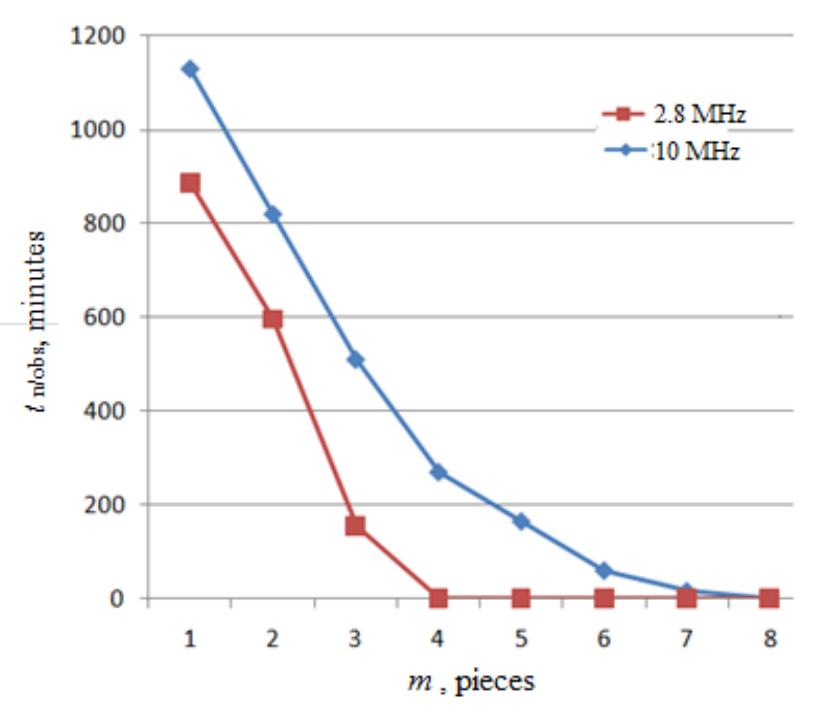

Fig. 6. The non-observance time $t_{n / \text { obs }}$ of the typical heating stand by the orbital constellation, including $m$ satellites, for the two boundary frequencies of the stand.

by a standard heater on conditions that a) one has no limitations in funding and time to deploy an orbit group, b) one can obtain no a priori data about heater's activity and c) one must provide non-stop monitoring of primary radiation generated by a heater, we obtain an orbit group consisting of eight satellites on high-elliptic orbit ("Molniya"type) with longitude correction in $34^{\circ}$.

\section{CONCLUSION}

Using the approach set forth in this paper one can synthesize orbit groups capable to monitor a) radiation generated by both any modern and designed in future earth-based heater and $b$ ) products of heater's activity.

\section{REFERENCES}

1. Cohen MB, Moore RC, Golkowski M, Lehtinen NG. ELF/VLF wave generation from the beating of two HF ionospheric heating sources. J. Geophys. Res., 2012, 117, A12310. doi: 10.1029/2012JA018140.

2. William F. Utlaut, Robert Cohen. Modifying the Ionosphere with Intense Radio Waves. Science, 1971, 174(4006):245.

3. Aleksashenko VA, Dvornikov SI, Dmitriev VG, Perunov YuM, Soloviov AA,
Tereschenko ED. Vestnik Akademii voennykh nauk, 2004, 3(8):131-137 (in Russ.).

4. Mingaleva GI, Mingalev VS. Response of the convecting high-latitude $\mathrm{F}$ layer to a powerful HF wave. Ann. Geophysicae, 1997, 15:1291.

5. Gurevich AV. Nonlinear effects in the ionosphere. Phys. Usp., 2007, 50(11):1091-1121; DOI: 10.1070/ PU2007v050n11ABEH006212.

6. Moore RC, Inan US, Bell TF, Kennedy EJ. ELF waves generated by modulated $\mathrm{HF}$ heating of the auroral electrojet and observed at a ground distance of $\sim 4400$ km. J. Geophys. Res., 2007, 112:A05309; doi: 10.1029/2006JA012063.

7. Barr R, Llanwyn Jones D, Rodger CJ. ELF and VLF radio waves. J. Atmos. Terr. Phys., 2000, 62:1689-1718.

8. Cohen MB. ELF/VLF phased array generation via frequency-matched steering of a continuous HF ionospheric heating beam. Ph. D. thesis. Palo Alto, Stanford university, 2009, 213 p.

9. Bell TF, Inan US, Helliwell RA. Nonducted coherent VLF waves and associated triggered emissions observed on the ISEE 1 satellite. $J$. Geophys. Res., 1981, 86:4649.

10. Kimura I, Matsumoto H, Mukai T, Hashimoto K, Bell TF, Inan US, Helliwell RA, Katsufrakis JP. EXOS-B/Siple Station VLF wave-particle interaction experiments: 1. General description and wave-particle correlations. J. Geophys. Res., 1983, 88:282.

11. Bell TF, Inan US, Kimura I, Matsumoto H, Mukai T, Hashimoto K. EXOS-B/Siple VLF wave-particle interaction experiments: 2 . Transmitter signals and associated emissions. J. Geophys. Res., 1983, 88:295.

12. Platino M, Inan US, Bell TF, Parrot M, Kennedy EJ. DEMETER observations of ELF waves injected with the HAARP HF transmitter. Geophys. Res. Lett., 2006, 33:L16101. doi:10.1029/2006GL026462. 
13. Murlaga AR. Solution of the inverse problem of monitoring from space of artificial controlled changes in the state of the ionosphere using the example of the HAARP stand. 9th Russ. scientific contest "Youth and future of aviation and cosmonautics". Moscow, MAI Publ., 2017:228-231 (in Russ.).

14. Murlaga AR. Monitoring of artificial changes in the ionosphere from the satellite: direct and inverse problem. Proc. XI Russ. conf. "Radiolocation and radio communication". Moscow, IRE RAS Publ., 2017:10-12 (in Russ.).

15. Gureev ED, Murlaga AR. Options for building a satellite system for monitoring the HAARP stand. Trudy MAI, 2013, 65 (in Russ.); http://mai.ru/upload/iblock/8 61/861 fe132228f739a4c673a57ecc9d195. pdf. 